\title{
Effects of probiotic yogurt consumption on metabolic factors in individuals with nonalcoholic fatty liver disease
}

\author{
S. Nabavi, ${ }^{*}$ M. Rafraf, ${ }^{* 1}$ M. H. Somi, $†$ A. Homayouni-Rad, $\ddagger$ and M. Asghari-Jafarabadi $\S^{2}$ \\ *Department of Community Nutrition, Faculty of Nutrition, \\ †Department of Internal Medicine, Faculty of Medicine, \\ †Department of Food Science and Technology, Faculty of Nutrition, and \\ $\S$ Health Management of National Center and Department of Statistics and Epidemiology, Faculty of Health, Tabriz University of Medical Sciences, \\ 51666-14711 Tabriz, Iran
}

\section{ABSTRACT}

The aim of this study was to investigate the effects of probiotic yogurt consumption on some metabolic factors in nonalcoholic fatty liver disease (NAFLD) patients. This double-blind, randomized, controlled clinical trial was conducted on 72 patients with NAFLD (33 males and 39 females) aged 23 to 63 yr. Subjects in the intervention group $(\mathrm{n}=36)$ consumed $300 \mathrm{~g} / \mathrm{d}$ of probiotic yogurt containing Lactobacillus acidophilus La5 and Bifidobacterium lactis Bb12 and those in the control group $(\mathrm{n}=36)$ consumed $300 \mathrm{~g} / \mathrm{d}$ of conventional yogurt for 8 wk. Fasting blood samples, anthropometric measurements, and dietary records $(24 \mathrm{~h} / \mathrm{d}$ for $3 \mathrm{~d}$ ) were collected at baseline and at the end of the trial. Probiotic yogurt consumption resulted in reductions of $4.67,5.42,4.1$, and $6.92 \%$ in serum levels of alanine aminotransferase, aspartate aminotransferase, total cholesterol, and low-density lipoprotein cholesterol, respectively, compared with control group. No significant changes were observed in levels of serum glucose, triglycerides, or high-density lipoprotein cholesterol in either group. Probiotic yogurt consumption improved hepatic enzymes, serum total cholesterol, and lowdensity lipoprotein cholesterol levels in studied subjects and might be useful in management of NAFLD risk factors.

Key words: nonalcoholic fatty liver disease, probiotic yogurt, metabolic factor

\section{INTRODUCTION}

Nonalcoholic fatty liver disease (NAFLD) is the most common form of chronic liver disease in the world and includes simple steatosis, nonalcoholic steatohepatitis, and fibrosis, which can finally develop to cirrhosis and

Received June 18, 2014.

Accepted August 26, 2014.

${ }^{1}$ Corresponding author: rafrafm@tbzmed.ac.ir

${ }^{2}$ Current address: Road Traffic Injury Research Center, Tabriz University of Medical Sciences, Tabriz, Iran. even hepatocellular carcinoma (Lomonaco et al., 2013). The prevalence of this disorder in general population screening with ultrasonography is reported to be 20 to $30 \%$ (Bellentani et al., 2010). Its prevalence in the in Iranian population is estimated to be about $33 \%$ (Adibi et al., 2008). Individuals with NAFLD have a higher mortality rate than healthy people (Wong, 2008). Longterm studies suggest that the most common factor in mortality of NAFLD patients is cardiovascular disease (Rafiq and Younossi, 2009). Diabetes and dyslipidemias, as comorbidities of NAFLD, predispose patients with NAFLD to cardiovascular disease (Clark et al., 2002). An increased levels of total cholesterol (TC) in blood is considered as a strong risk factor for coronary heart disease (Nguyen et al., 2007). In general, each 1\% reduction of the cholesterol level causes a $2.3 \%$ decrease in coronary-related risks. Therefore, lowering the cholesterol level can be an excellent solution in reducing cardiovascular diseases mortality (Baroutkoub et al., 2010). Dyslipidemia occurs in 20 to $80 \%$ of individuals with NAFLD (Day, 2004).

Current treatments for NAFLD include changes in diet, increased physical activity, medication, and surgery (Gill and Wu, 2006). In recent years, probiotics have been discussed as a potential alternative in the treatment of various diseases, including NAFLD (Lata et al., 2011).

The World Health Organization describes probiotics as live microorganisms that generate a health benefit on the host when administered in sufficient amounts (Metchnikoff, 1907; reprinted 2004). Metchnikoff, a Nobel Prize winner, was the first to propose probiotics as a useful factor in human health (FAO-WHO, 2001). The reported prophylactic and therapeutic effects of these microorganisms are as follows: balancing the intestinal microflora; reducing cholesterol levels; improving hypertension, diabetes, lactose intolerance, gastrointestinal diseases; promoting immune system; and lowering the risk of different types of cancer (Viana et al., 2007; Goldin and Gorbach, 2008; Kaur et al., 2009; Lye et al., 2009). 
It has been claimed that consumption of probiotic products significantly decreases serum cholesterol (Niazmand et al., 2010). Therefore, many studies have been performed to clarify the effect of fermented dairy products on serum cholesterol, but the cholesterol-lowering effects of probiotics have not yet been established (Xiao et al., 2003; Lewis and Burmeister, 2005; Greany et al., 2008; Ataie-Jafari et al., 2009; Sadrzadeh-Yeganeh et al., 2010). In most of these investigations, reductions in serum cholesterol were reported only in humans who consumed very high doses of fermented dairy products. Other interventions using "normal" doses of fermented milk product were unsuccessful in confirming such findings. Nevertheless, the possible advantages of probiotics dairy products on serum lipid profile remain under debate.

As mentioned above, hepatic lipid homeostasis is disordered in NAFLD (Kneeman et al., 2012). To our knowledge, no reports are available on the effects of probiotic products, including yogurt, on metabolic status in NAFLD patients; hence, the current study was designed to investigate the effects of probiotic yogurt containing Lactobacillus acidophilus La5 and Bifidobacterium lactis $\mathrm{Bb} 12$, compared with conventional yogurt, on metabolic factors, including liver enzymes, fasting blood sugar (FBS), and serum lipid profile in individuals with NAFLD.

\section{MATERIALS AND METHODS}

\section{Subjects}

Seventy-two NAFLD subjects, aged 23 to $63 \mathrm{yr}$, with body mass index (BMI) ranging from 25 to $40 \mathrm{~kg} /$ $\mathrm{m}^{2}$, were recruited in this double-blinded, randomized, controlled clinical trial from the Shykhoraeis polyclinic (Tabriz, Iran).

Recruitment was done via referral from a gastroenterologist. All participants had been diagnosed as new cases of NAFLD. The diagnostic criterion for NAFLD and its degree was ultrasonography. Exclusion criteria were presence of kidney disease, another type of liver disease, hepatitis B or C, inflammatory intestinal disease, thyroid disorders, immunodeficiency diseases, Wilson disease, or hemochromatosis; lactose intolerance; using tobacco or alcohol; taking nutritional supplements within the previous $3 \mathrm{wk}$ or during the 8-wk study period; receiving cholesterol-lowering medication, estrogen, progesterone, or diuretics; being pregnant or breast feeding; and consuming probiotic yogurt or any other probiotic products within the previous 2 mo.

Sample size was determined based on primary information obtained from the study by Ejtahed et al. (2011) for FBS. Considering $\alpha=0.05$ and a power of
$80 \%$, the sample size was computed as $30.49(\approx 31)$ per group (Pocock, 1983). This number was increased to 36 per group to accommodate the anticipated dropout rate.

\section{Study Design and Measurements}

This study was conducted on individuals with NAFLD (33 males and 39 females). Participants were randomly assigned into 2 groups using a block randomization procedure of size 4 . The random sequence was generated by the study statistician using random allocation software. Randomization was stratified according to age, sex, and BMI. In total, 36 patients were entered in each group. During the 1-wk adjustment period, all patients refrained from eating yogurt or any other fermented food. Over $8 \mathrm{wk}$, the intervention and control groups consumed $300 \mathrm{~g}$ daily of probiotic or conventional yogurt, respectively. All subjects were told not to alter their usual dietary habits or lifestyle and to avoid consuming any yogurt other than that provided to them by the researchers throughout the 8 -wk trial. The volunteers were also instructed to keep the yogurt under refrigeration.

The assignment of groups was blinded to investigators and subjects. In addition, the statistician was not aware of the allocation of participants to intervention and control groups. Probiotic and conventional yogurt containers were identical in appearance. Every week, the subjects would receive a week's supply of their probiotic or conventional yogurts (containing $2.5 \%$ fat) from researchers. Through weekly follow-ups by phone interview, compliance with the yogurt consumption was monitored once per week.

Recording of food consumption information (through 24-h dietary recall for $3 \mathrm{~d}$ ), anthropometric measurements, and collection of fasting blood samples were done at the beginning and end of the trial. The 24-h dietary recall comprised 1 weekend day and 2 nonconsecutive weekdays. Three-day averages of energy and macronutrient intakes were analyzed by using Nutritionist 4 software (First Databank Inc., Hearst Corp., San Bruno, CA). All data were entered by trained dietitians.

Body weight was measured using a scale (Seca, Hamburg, Germany) with 0.5-kg accuracy while participants were without shoes and wearing light clothing. A tape was used to measure height with 0.5 -cm accuracy while participants were without shoes. Body mass index was calculated using weight and height measurements $(\mathrm{kg} /$ $\left.\mathrm{m}^{2}\right)$.

Blood samples were collected in the morning after a 12-h overnight fast. The blood sample was drawn from the antecubital vein in the arm. The serum samples 
were separated from whole blood by centrifugation at $2,606.8 \times g$ for $10 \mathrm{~min}$ at $4^{\circ} \mathrm{C}$ (Beckman Avanti J-25; Beckman Coulter, Brea, CA). The serum samples were frozen immediately at $-70^{\circ} \mathrm{C}$ until assay. Blood samples were analyzed at the Drug Applied Research Center (Tabriz University of Medical Sciences, Tabriz, Iran). Alanine aminotransferase (ALT) and aspartate aminotransferase (AST) were measured by using Bio Systems kits (Barcelona, Spain) and autoanalyzer set (Hitachi 911, Depok, Indonesia). Fasting blood glucose was measured using the standard enzymatic method with a Parsazmun kit (Karaj, Iran). Serum concentrations of TC, triglyceride (TG), and high-density lipoprotein cholesterol (HDL-C) were measured using the standard enzymatic methods with commercially available Parsazmun kits; TC was assayed using cholesterol esterase and cholesterol oxidase method, and TG was assayed by using glycerol phosphate oxidase. The concentration of HDL-C was measured after precipitation of the apolipoprotein B-containing lipoproteins. Lowdensity lipoprotein cholesterol concentration (LDL-C) was determined by the Friedewald formula (Friedewald et al., 1972).

This study was approved by the ethics committee at Tabriz University of Medical Sciences and was conducted according to the principles laid down in the Declaration of Helsinki. Prior to the study, purpose and methodology of the study were fully explained to the participants by the researchers, and written informed consent was obtained from all participants.

\section{Intervention}

Both probiotic and conventional yogurts contained Lactobacillus bulgaricus and Streptococcus thermophilus. The probiotic yogurt was also enriched by adding direct-vat-set cultures of $B$. lactis $\mathrm{Bb} 12$ and L. acidophilus La5 (Chr. Hansen, Hørsholm, Denmark). The yogurts were produced weekly and distributed to the participants. Time of distribution of yogurts was $1 \mathrm{~d}$ after manufacture, and yogurts were microbiologically analyzed every week.

Microbiological analyses of the probiotic yogurts showed that the average counts of L. acidophilus La5 and B. lactis $\mathrm{Bb} 12$ on $\mathrm{d} 1$ were $6.46 \times 10^{6}$ and 4.97 $\times 10^{6} \mathrm{cfu} / \mathrm{g}$, respectively. Probiotic yogurts contained $2.39 \times 10^{6} \mathrm{cfu} / \mathrm{g}$ of L. acidophilus La5 and $2.08 \times 10^{6}$ $\mathrm{cfu} / \mathrm{g}$ of B. lactis Bb12 on d 7. Both probiotic bacteria showed a steady survival rate during the 7-d storage time at an average concentration of $4.42 \times 10^{6} \mathrm{cfu} / \mathrm{g}$ and $3.85 \times 10^{6} \mathrm{cfu} / \mathrm{g}$, respectively. The probiotic and conventional yogurt containers were identical and the organoleptic characteristics (e.g., taste and appearance) of the yogurts were similar. Both types of yogurt could be found in Iranian markets, but the yogurts were specially prepared for this study by the Pegah Dairy Industries Co. (Tabriz, Iran).

\section{Statistical Analyses}

The experimental data were analyzed by using SPSS software (version 16; SPSS Inc., Chicago, IL), and results were expressed as mean \pm standard deviation or number (\%). The normality of the distribution of variables was checked by the Kolmogorov-Smirnov test. For ALT and AST with abnormal distribution, analyses were performed after log transformation. The background characteristics and nutrient intakes of participants in the 2 groups were compared using independent sample $t$-tests and chi-squared test.

Analysis of covariance was used to identify any differences between the 2 groups after intervention, adjusting for baseline measurements and confounders (BMI and energy changes during study). The changes in anthropometric measurements, dietary intakes, and blood lipid parameters of the participants between the beginning and end of the trial were compared by paired sample $t$-tests. Statistical significance was declared at $P<0.05$ (Zar, 1998).

\section{RESULTS}

All subjects completed the study. Participants demonstrated good compliance with yogurt consumption and no adverse effects or symptoms were reported. Table 1 presents the general, clinical, and anthropometric characteristics of the participants. We observed no significant differences between the 2 groups in terms of age, sex, anthropometric measurements, or grade of fatty liver at baseline. Mean BW and BMI significantly decreased in the probiotic yogurt group compared with conventional yogurt group by the end of the study ( $P$ $<0.01)$.

Daily dietary intakes of participants throughout the study are shown in Table 2. We detected no significant differences in energy or macronutrient intakes between the 2 groups at baseline. Mean protein intake increased significantly in both groups compared with baseline values at the end of study $(P<0.05)$. Changes in energy and other macronutrient intakes were not significant in either group.

Metabolic parameters of subjects at baseline and after the 8-wk intervention are presented in Table 3. We detected no statistically significant differences between the 2 groups in terms of serum liver enzymes or glucose and lipid levels at baseline. Results of analysis of covariance showed statistically significant differences between the 2 studied groups in serum ALT and AST (both 
Table 1. General, anthropometric, and clinical characteristics of patients with nonalcoholic fatty liver disease

\begin{tabular}{|c|c|c|c|}
\hline Item & $\begin{array}{l}\text { Conventional yogurt } \\
\qquad(\mathrm{n}=36)\end{array}$ & $\begin{array}{l}\text { Probiotic yogurt } \\
\qquad(\mathrm{n}=36)\end{array}$ & $P$-value \\
\hline \multicolumn{4}{|l|}{ Age, yr } \\
\hline Mean (SD) & $44.05(8.14)$ & $42.75(8.72)$ & \multirow[t]{2}{*}{0.51} \\
\hline Range & $27-58$ & $23-63$ & \\
\hline \multicolumn{4}{|l|}{ Sex, no. (\%) } \\
\hline Male & $18(50 \%)$ & $17(48.2 \%)$ & \multirow[t]{2}{*}{0.81} \\
\hline Female & $18(50 \%)$ & $19(52.8 \%)$ & \\
\hline \multicolumn{4}{|c|}{ Grade of fatty liver } \\
\hline \multicolumn{4}{|c|}{ Grade 1, no. (\%) } \\
\hline Baseline & $21(58.3 \%)$ & $20(55.6 \%)$ & \\
\hline Intervention & $21(58.3 \%)$ & $19(52.8 \%)$ & \\
\hline \multicolumn{4}{|c|}{ Grade 2, no. $(\%)$} \\
\hline Baseline & $12(33.3 \%)$ & $12(33.3 \%)$ & \\
\hline Intervention & $7(19.4 \%)$ & $5(13.9 \%)$ & \\
\hline \multicolumn{4}{|c|}{ Grade 3, no. (\%) } \\
\hline Baseline & $3(8.3 \%)$ & $4(11.1 \%)$ & \\
\hline Intervention & $2(5.6 \%)$ & $1(2.8 \%)$ & \\
\hline \multicolumn{4}{|c|}{ Mean (SD) BW, kg } \\
\hline Baseline & $86.23(12.23)$ & $84.32(13.2)$ & \\
\hline Intervention & $85.98(11.94)$ & $82.58 *(13.33)$ & \\
\hline \multicolumn{4}{|c|}{ Mean (SD) body mass index. $\mathrm{kg} / \mathrm{m}^{2}$} \\
\hline Baseline & $31.4(3.6)$ & $30.1(3.61)$ & \\
\hline Intervention & $31.29(3.7)$ & $29.48^{*}(3.7)$ & \\
\hline
\end{tabular}

*Significant difference between groups after intervention $(P<0.05$; independent sample $t$-test $)$.

$P<0.02)$, TC $(P<0.001)$, and LDL-C $(P<0.01)$ when values were adjusted for BMI, energy intake, and baseline values at the end of study. Changes in serum glucose, TG, and HDL-C levels were not significant.

Probiotic yogurt consumption resulted in reductions of $4.67,5.42,4.1$, and $6.92 \%$ in serum levels of ALT, AST, TC, and LDL-C, respectively, compared with these variables in the conventional yogurt group. Probiotic yogurt consumption also caused significant reductions in serum ALT, LDL-C (both $P<0.005$ ), $\mathrm{AST}$, and TC $(P<0.001)$ concentrations compared with the baseline values. Serum glucose, TG, and HDL$\mathrm{C}$ concentrations remained unchanged in the probiotic group after intervention. Serum levels of HDL-C increased significantly in the control group during the study $(P<0.05)$.

\section{DISCUSSION}

Hepatic transaminases, including ALT and AST, are indicators of hepatocellular injury. The serum concentrations of these enzymes in individuals with NAFLD range from slightly above the upper limit of normal $(<31 \mathrm{IU} / \mathrm{L}$ ALT or AST in women and $<41 \mathrm{IU} / \mathrm{L}$ ALT and $<37 \mathrm{IU} / \mathrm{L}$ AST in men) to 10 times higher (Nanda, 2004; Roberts, 2005); NAFLD may also be present with normal or fluctuating AST and ALT levels (Rafiq and Younossi, 2009).

In present study, 30.6 and $34.7 \%$ of subjects had elevated levels of ALT and AST, respectively, at baseline. Our study revealed that consumption of the probiotic yogurt containing L. acidophilus La5 and B. lactis Bb12 for 8 wk resulted in significant reductions in serum ALT

Table 2. Dietary intake of patients with nonalcoholic fatty liver disease throughout the study ${ }^{1}$

\begin{tabular}{llcc}
\hline Variable & $\begin{array}{l}\text { Measurement } \\
\text { period }\end{array}$ & $\begin{array}{c}\text { Conventional yogurt } \\
(\mathrm{n}=36)\end{array}$ & $\begin{array}{c}\text { Probiotic yogurt } \\
(\mathrm{n}=36)\end{array}$ \\
\hline Energy, kcal/d & Before & $1,922.85 \pm 122.99$ & $1,914.32 \pm 96.49$ \\
Carbohydrate, g/d & After & $2,072.46 \pm 114.85$ & $1,934.77 \pm 99.11$ \\
& Before & $282.09 \pm 19.59$ & $288.01 \pm 15.92$ \\
Protein, g/d & After & $304.96 \pm 19.39$ & $274.1 \pm 15.48$ \\
& Before & $73.72 \pm 6.08$ & $71.55 \pm 4.25$ \\
Total fat, g/d & After & $84.11 \pm 6.00^{*}$ & $84.62 \pm 6.32^{*}$ \\
& Before & $57.89 \pm 4.13$ & $58.41 \pm 3.78$ \\
& After & $58.24 \pm 3.61$ & $57.64 \pm 3.47$ \\
${ }^{1}$ Data are presented as means \pm SD. & \\
${ }^{*}$ Significant difference within group throughout the study $(P<0.05$, paired samples $t$-test $)$.
\end{tabular}


Table 3. Serum metabolic factors of patients with nonalcoholic fatty liver disease through the study

\begin{tabular}{|c|c|c|}
\hline Metabolic factor ${ }^{1}$ & $\begin{array}{l}\text { Conventional yogurt } \\
\qquad(\mathrm{n}=36)\end{array}$ & $\begin{array}{l}\text { Probiotic yogurt } \\
\quad(\mathrm{n}=36)\end{array}$ \\
\hline \multicolumn{3}{|l|}{$\mathrm{ALT}^{2}{ }^{\mathrm{IU}} / \mathrm{L}$} \\
\hline Baseline & $25.5(20-37)$ & $31.5(21-49.59)$ \\
\hline Intervention & $24.5(19.25-34.5)$ & $25.5(20-40.25) * \dagger$ \\
\hline Mean (SD) percentage change & $3.71(26.14)$ & $-13.06(27.6)$ \\
\hline Abnormal cases at baseline, no. (\%) & $9(25 \%)$ & $13(36.11 \%)$ \\
\hline Abnormal cases after intervention, no. (\%) & $9(25 \%)$ & $10(27.77 \%)$ \\
\hline \multicolumn{3}{|l|}{$\mathrm{AST},{ }^{2} \mathrm{IU} / \mathrm{L}$} \\
\hline Baseline & $26(20.25-36.5)$ & $32.5(24.25-46.5)$ \\
\hline Intervention & $25(22-35)$ & $27.5(21.25-36.75) * \dagger$ \\
\hline Mean (SD) percentage change & $3.01(25.41)$ & $-13.87(21.88)$ \\
\hline Abnormal cases at baseline, no. (\%) & $9(25 \%)$ & $15(41.66 \%)$ \\
\hline Abnormal cases after intervention, no. (\%) & $8(22.22 \%)$ & $11(30.55 \%)$ \\
\hline \multicolumn{3}{|l|}{ Glucose, $\mathrm{mg} / \mathrm{dL}$} \\
\hline Baseline & $87.11 \pm 13.7$ & $90.11 \pm 9.62$ \\
\hline Intervention & $89.91 \pm 12.22$ & $89.25 \pm 9.54$ \\
\hline Mean (SD) percentage change & $4.04(12.14)$ & $-0.6(8.64)$ \\
\hline Abnormal cases at baseline, no. (\%) & $3(8.33 \%)$ & $3(8.33 \%)$ \\
\hline Abnormal cases after intervention, no. (\%) & $2(5.55 \%)$ & $2(5.55 \%)$ \\
\hline \multicolumn{3}{|l|}{ Total cholesterol, mg/d } \\
\hline Baseline & $198.63 \pm 31.87$ & $196.55 \pm 39.18$ \\
\hline Intervention & $202.88 \pm 33.53$ & $172.61 \pm 42.6^{*} \dagger$ \\
\hline Mean (SD) percentage change & $3.02(14.11)$ & $-11.23(16.77)$ \\
\hline Abnormal cases at baseline, no. (\%) & $14(38.88 \%)$ & $18(50 \%)$ \\
\hline Abnormal cases after intervention, no. (\%) & $14(38.88 \%)$ & $8(22.22 \%)$ \\
\hline \multicolumn{3}{|l|}{ Triglyceride, $\mathrm{mg} / \mathrm{d}$} \\
\hline Baseline & $197.5 \pm 77.09$ & $194.16 \pm 63.89$ \\
\hline Intervention & $206.02 \pm 79.39$ & $172.91 \pm 68.59$ \\
\hline Mean (SD) percentage change & $5.77(27.32)$ & $-5.36(41.69)$ \\
\hline Abnormal cases at baseline, no. (\%) & $25(69.44 \%)$ & $25(69.44 \%)$ \\
\hline Abnormal cases after intervention, no. (\%) & $25(69.44 \%)$ & $20(55.55 \%)$ \\
\hline \multicolumn{3}{|l|}{$\mathrm{LDL}-\mathrm{C}, \mathrm{mg} / \mathrm{d}$} \\
\hline Baseline & $111.3 \pm 29.69$ & $120.08 \pm 35.22$ \\
\hline Intervention & $110.03 \pm 26.94$ & $99.83 \pm 21.79^{*} \dagger$ \\
\hline Mean (SD) percentage change & $2.13(28.3)$ & $-15.98(33.79)$ \\
\hline Abnormal cases at baseline, no. (\%) & $10(27.77 \%)$ & $10(27.77 \%)$ \\
\hline Abnormal cases after intervention, no. (\%) & $8(22.22 \%)$ & $6(16.66 \%)$ \\
\hline \multicolumn{3}{|l|}{ HDL-C, mg/d } \\
\hline Baseline & $47.83 \pm 9.61$ & $47.63 \pm 10.88$ \\
\hline Intervention & $50.84 \pm 9.84 \dagger$ & $49.19 \pm 12.2$ \\
\hline Mean (SD) percentage change & $8.30(20.44)$ & $3.82(15.75)$ \\
\hline Abnormal cases at baseline, no. (\%) & $14(38.88 \%)$ & $14(38.88 \%)$ \\
\hline Abnormal cases after intervention, no. (\%) & $10(27.77 \%)$ & $16(44.44 \%)$ \\
\hline
\end{tabular}

${ }^{1} \mathrm{ALT}=$ alanine aminotransferase; AST $=$ aspartate aminotransferase; LDL-C = low-density lipoprotein cholesterol; HDL-C = high-density lipoprotein cholesterol. ALT and AST are presented as median (25th percentile -75 th percentile); other data are expressed as means \pm SD.

* Significant difference between conventional and probiotic yogurt groups after intervention $(P<0.05$ for ALT and AST, $P<0.001$ for total cholesterol, and $P<0.01$ for LDL-C; analysis of covariance (for ALT and AST after log-transformation).

$\dagger$ Significant difference $(P<0.05)$ within groups throughout the study; paired $t$-test (for ALT and AST after log-transformation).

and AST in NAFLD patients. In accordance with results of this trial, Aller et al. (2011), in a double-blind, randomized clinical pilot study on NAFLD patients, found that treatment for 3 mo with a tablet containing Lactobacillus bulgaricus and Streptococcus thermophilus reduced serum ALT and AST levels. Significant reductions in serum AST level were observed in a study by Malaguarnera et al. (2007), where subjects with NAFLD received a capsule containing Bifidobacterium longum with fructo-oligosaccharides for $24 \mathrm{wk}$. Loguercio et al.
(2002), in a pilot study, reported that intervention for 2 mo with several species of Lactobacillus (acidophilus, bifidus, rhamnosus, plantarum, salivarius, bulgaricus, lactis, casei, and breve) mixed with prebiotic fructooligosaccharide and some vitamins decreased serum concentrations of ALT in patients with this NAFLD. Results of several studies on NAFLD animal models also indicated useful effects of probiotics on serum ALT value (Li et al., 2003; Karahan et al., 2012; Bhathena et al., 2013). In 2 studies in rats, serum AST (Esposito et 
al., 2009) and serum AST and ALT (Paik et al., 2005) were significantly reduced after feeding probiotics. It was suggested that probiotic bacteria were capable of reducing the effect of pathogenic bacteria on NAFLD development by exclusion or inhibition and by producing short-chain fatty acids as antimicrobial factors. However, several studies have not report any effects of probiotics on liver enzymes (Li et al., 2003; Esposito et al., 2009; Karahan et al., 2011; Bhathena et al., 2013).

Based on our results (Table 3), no significant changes in FBS were seen in individuals at the end of the study in either group. Similar to our results, Aller et al. (2011), in a pilot study on individuals with NAFLD, did not find significant changes in blood glucose after a 3-mo intervention using Lactobacillus bulgaricus and Streptococcus thermophilus. In the study of Bhathena et al. (2013), treatment with Lactobacillus fermentum for $12 \mathrm{wk}$ had no significant effect on glucose levels in hamsters. On the other hand, in some animal and human studies, probiotics improved serum elevated glucose (Paik et al., 2005; Yadav et al., 2008; Ejtahed et al., 2011). It should be noted that, in our study, more than $90 \%$ of subjects in both groups had normal FBS at baseline. Therefore, no significant changes in this variable were expected.

Individuals with NAFLD often have dyslipidemia, which is characterized by increased serum TG and LDL-C levels and low HDL-C levels (Chatrath et al., 2012). Such conditions existed in $71 \%$ of our subjects at baseline. According to our results (Table 3), probiotic yogurt intake lowered serum TC and LDL-C concentrations significantly in the intervention group compared with the control group. Our results are in agreement with the findings of some animal and human studies. In the study by Paik et al. (2005), a probiotic containing Bacillus polyfermenticus SCD reduced plasma LDL and cholesterol levels in NAFLD rats. In Malaguarnera et al. (2012), serum LDL-C levels decreased during 24-wk consumption of Bifidobacterium longum with fructooligosaccharides by individuals with nonalcoholic steatohepatitis. In a study on NAFLD rats, a diet with Lactobacillus plantarum MA2 decreased plasma TC and TG (Wang et al., 2009).

Ejtahed et al. (2011) reported that daily consumption of $300 \mathrm{~g}$ of yogurt containing L. acidophilus La5 and B. lactis Bb12 by individuals with type 2 diabetes mellitus led to decreases of 4.54 and $7.45 \%$ in serum TC and LDL-C levels, respectively. In another study, in hypercholesterolemic humans, the concentration of TC decreased by $2.9 \%$ in response to consumption of $200 \mathrm{~g}$ of yogurt containing L. acidophilus L1 (Anderson and Gilliland, 1999). In a study by Ataie-Jafari et al. (2009), a significant decline in serum TC was observed with consumption of $300 \mathrm{~g}$ of probiotic yogurt contain- ing $L$. acidophilus and B. lactis daily in hypercholesterolemic people. In another study, Baroutkoub et al. (2010) showed that consumption of probiotic yogurt containing L. acidophilus and bifidobacteria caused a significant decrease in plasma TC and LDL-C concentrations after 6 wk. Fabian and Elmadfa (2006) and Sadrzadeh- Yeganeh et al. (2010) demonstrated that both probiotic and conventional yogurts have positive effects on the lipid profiles of healthy women.

Several mechanisms for cholesterol-lowering effects of probiotics have been proposed. In vitro experiments have demonstrated that intestinal lactic acid bacteria can assimilate and bind cholesterol, as well as bile acids, within the bacterial cells (Zhuang et al., 2012). As a result, dietary cholesterol would be less available for absorption in the intestine and TC would be decreased. Deconjugation of bile acids by bile salt hydrolase is thought to be another mechanism for serum lipid improvement; it might interfere with the enterohepatic circulation of bile salts. Whereas cholesterol is the precursor for the synthesis of new bile acids, deconjugation of bile acids by bile salt hydrolase would lead to a decrease in serum cholesterol concentration (Begley et al., 2006; Ooi and Liong, 2010).

The production of short-chain fatty acids by probiotics during fermentation inhibits hepatic cholesterol synthesis. The enzyme 3-hydroxy-3-methyl-glutaryl-CoA (HMG-CoA) reductase, which catalyzes the reduction of HMG-CoA to mevalonate, is the rate-determining enzyme of the cholesterol biosynthetic pathway and it is regarded as a therapeutic target for the treatment of hypercholesterolemia. Recent research has indicated that probiotics might inhibit expression of $\mathrm{HMG}-\mathrm{CoA}$ reductase (Homayouni, 2008; Ooi and Liong, 2010; Zhuang et al., 2012). Coprostanol is a product of cholesterol that is excreted in the feces; it also reduces cholesterol absorption and lowers blood cholesterol levels. Coprostanol production is increased in the presence of probiotics (Ooi and Liong, 2010; Kumar et al., 2012).

Some studies do not support the cholesterol-lowering potential of probiotics when used in the form of capsules (Lewis and Burmeister, 2005; Greany et al., 2008; Hatakka et al., 2008; Aller et al., 2011). In the study of Lewis and Burmeister (2005), insufficient time for metabolic activation of probiotic bacteria in capsules might explain the lack of effect in lowering serum cholesterol. It has been suggested that dairy products are more effective media for administrating probiotics compared with probiotic supplements. Our results were similar to those of different clinical studies (Anderson and Gilliland, 1999; Fabian and Elmadfa, 2006; Ataie-Jafari et al., 2009; Baroutkoub et al., 2010; Ejtahed et al., 2011; Sadrzadeh- Yeganeh et al., 2010) and confirmed that probiotics administered in yogurt had beneficial effects 
on serum LDL-C and TC levels in the studied subjects. These effects may be also clinically useful (the number needed to treat was calculated as $33.33,25,10$, and 25 for ALT, AST, TC, and LDL-C, respectively).

In addition to the mechanisms mentioned above, sphingolipids in yogurts and in cell membranes of probiotic bacteria are effective in cholesterol metabolism and transport, by reducing absorption and increasing excretion (Kiessling et al., 2002). It is possible that probiotics can decrease serum TG such as cholesterol in a similar way as probiotics decrease blood cholesterol as noted herein.

Based on our results, we did not find significant changes in serum TG and HDL-C levels in the probiotic group compared with the control group. In agreement with our results, Ejtahed et al. (2011) did not observe significant changes in serum TG and HDL-C levels in subjects with diabetes with a design similar to that of the present study. Sadrzadeh-Yeganeh et al. (2010) also did not report significant difference in TG in healthy women after consumption of probiotic yogurt for $6 \mathrm{wk}$. In the study of Baroutkoub et al. (2010), consumption of probiotic yogurt for 6 wk did not change serum HDL$\mathrm{C}$ levels in hypercholesterolemic individuals. Lewis and Burmeister (2005) conducted a randomized, placebocontrolled, double-blind crossover study to determine the effect of capsules containing L. acidophilus on lipid profiles of volunteers with elevated cholesterol levels for $6 \mathrm{wk}$. They found no significant changes in plasma HDL-C or TC levels. However, some studies documented that probiotics improved HDL-C concentration or decreased TG levels (Kawase et al., 2000; Naruszewicz et al., 2002; Baroutkoub et al., 2010). These conflicting findings could be due in part to varying strains and doses of probiotics, differences in duration of treatment periods, sample size, and clinical characteristics of participants, or different experimental designs (Ooi and Liong, 2010).

The limitations of our study include the absence of a control group that consumed no yogurt and use of the ultrasonography technique instead of biopsy as the diagnostic criterion for NAFLD. Investigations with longer duration and a control group without yogurt intake are needed for better confirmation of the possible effects of probiotic yogurt on metabolic status of individuals with NAFLD.

\section{CONCLUSIONS}

Consumption of probiotic yogurt containing $L$. acidophilus La5 and B. lactis Bb12 had beneficial effects on hepatic enzymes and serum TC and LDL-C levels in the studied subjects. Probiotic yogurt might be useful in management of NAFLD risk factors.

\section{ACKNOWLEDGMENTS}

We thank the Research Vice-Chancellor and Nutrition Research Center of Tabriz University of Medical Sciences (Tabriz, Iran) for the financial support; Pegah Dairy Industries Co. (Tabriz, Iran) for supplying the probiotic and conventional yogurts; and the patients who participated in this study.

\section{REFERENCES}

Adibi, A., A. Hekmatnia, and A. Shayganfar. 2008. Ultrasound screening of nonalcoholic fatty liver in Isfahan Medical University personnel. Iran. J. Radiol. 5:1.

Aller, R., D. A. De Luis, O. Izaola, R. Conde, M. Gonzalez Sagrado, D. Primo, B. De La Fuente, and J. Gonzalez. 2011. Effect of a probiotic on liver aminotransferases in nonalcoholic fatty liver disease patients: A double blind randomized clinical trial. Eur. Rev. Med. Pharmacol. Sci. 15:1090-1095.

Anderson, J. W., and S. E. Gilliland. 1999. Effect of fermented milk (yogurt) containing Lactobacillus acidophilus L1 on serum cholesterol in hypercholesterolemic humans. J. Am. Coll. Nutr. 18:4350 .

Ataie-Jafari, A., B. Larijani, H. Alavi Majd, and F. Tahbaz. 2009 Cholesterol-lowering effect of probiotic yogurt in comparison with ordinary yogurt in mildly to moderately hypercholesterolemic subjects. Ann. Nutr. Metab. 54:22-27.

Baroutkoub, A., M. Roushan Zamir, B. Razmik, H. Julayi, Z. Sohrabi, S. M. Mazloomi, and M. H. Eskandari. 2010. Effects of probiotic yoghurt consumption on the serum cholesterol levels in hypercholesteremic cases in Shiraz, Southern Iran. Sci. Res. Essays 5:2206-2209.

Begley, M., C. Hill, and C. G. M. Gahan. 2006. Bile salt hydrolase activity in probiotics. Appl. Environ. Microbiol. 72:1729-1738.

Bellentani, S., F. Scaglioni, M. Marino, and G. Bedogni. 2010. Epidemiology of non-alcoholic fatty liver disease. Dig. Dis. 28:155-161. http://dx.doi.org/10.1159/000282080.

Bhathena, J., C. Martoni, A. Kulamarva, C. Tomaro-Duchesneau, M. Malhotra, A. Paul, A. M. Urbanska, and S. Prakash. 2013. Oral probiotic microcapsule formulation ameliorates non-alcoholic fatty liver disease in Bio $F_{1} B$ Golden Syrian hamsters. PLoS ONE 8:e58394 http://dx.doi.org/10.1371/journal.pone.0058394.

Chatrath, H., R. Vuppalanchi, and N. Chalasani. 2012. Dyslipidemia in patients with nonalcoholic fatty liver disease. Semin. Liver Dis. 32:22-29. http://dx.doi.org/10.1055/s-0032-1306423.

Clark, J. M., F. Brancati, and A. Diehl. 2002. Nonalcoholic fatty liver disease. Gastroenterology 122:1649-1657. http://dx.doi. org/10.1053/gast.2002.33573.

Day, C. P. 2004. Non-alcoholic fatty liver disease. Pages 393-404 in Evidence-Based Gastroenterology.

Ejtahed, H. S., J. Mohtadi-Nia, A. Homayouni-Rad, M. Niafar, M. Asghari-Jafarabadi, V. Mofid, and A. Akbarian-Moghari. 2011. Effect of probiotic yogurt containing Lactobacillus acidophilus and Bifidobacterium lactis on lipid profile in individuals with type 2 diabetes mellitus. J. Dairy Sci. 94:3288-3294. http://dx.doi. org $/ 10.3168 /$ jds.2010-4128.

Esposito, E., A. Iacono, G. Bianco, G. Autore, S. Cuzzocrea, P. Vajro, R. B. Canani, A. Calignano, G. M. Raso, and R. Meli. 2009. Probiotics reduce the inflammatory response induced by a high-fat diet in the liver of young rats. J. Nutr. 139:905-911. http://dx.doi. org/10.3945/jn.108.101808.

Fabian, E., and I. Elmadfa. 2006. Influence of daily consumption of probiotic and conventional yoghurt on the plasma lipid profile in young healthy women. Ann. Nutr. Metab. 50:387-393.

FAO-WHO (Food and Agriculture Organization of the United Nations/World Health Organization). 2001. WHO Expert consultation on evaluation of health and nutritional properties of probiotics in food including powder milk with live lactic acid bacteria. 
Accessed Sep. 8, 2014. ftp://ftp.fao.org/docrep/fao/009/a0512e/ a0512e00.pdf.

Friedewald, W. T., R. I. Levy, and D. S. Fredrickson. 1972. Estimation of the concentration of low-density lipoprotein cholesterol in plasma, without use of the preparative ultracentrifuge. Clin. Chem. 18:499-502.

Gill, H. K., and G. Y. Wu. 2006. Non-alcoholic fatty liver disease and the metabolic syndrome: Effects of weight loss and a review of popular diets. Are low carbohydrate diets the answer? World J. Gastroenterol. 12:345-353.

Goldin, B. R., and S. L. Gorbach. 2008. Clinical indications for probiotics: an overview. Clin. Infect. Dis. 46(Suppl. 2):S96-100. http://dx.doi.org/10.1086/523333.

Greany, K. A., M. J. L. Bonorden, J. M. Hamilton-Reeves, M. H. McMullen, K. E. Wangen, W. R. Phipps, J. Feirtag, W. Thomas, and M. S. Kurzer. 2008. Probiotic capsules do not lower plasma lipids in young women and men. Eur. J. Clin. Nutr. 62:232-237.

Hatakka, K., M. Mutanen, R. Holma, M. Saxelin, and R. Korpela. 2008. Lactobacillus rhamnosus LC705 together with Propionibacterium freudenreichii ssp. shermanii JS administered in capsules is ineffective in lowering serum lipids. J. Am. Coll. Nutr. 27:441447.

Homayouni, A. 2008. Therapeutical Effects of Functional Probiotic, Prebiotic and Symbiotic Foods. Tabriz University of Medical Sciences, Tabriz, Iran.

Karahan, N., M. Işler, A. Koyu, A. G. Karahan, G. Başyığıt Kiliç, I. M. Cırış, R. Sütçü, I. Onaran, H. Cam, and M. Keskın. 2012. Effects of probiotics on methionine choline-deficient diet-induced steatohepatitis in rats. Turk. J. Gastroenterol. 23:110-121.

Kaur, I. P., A. Kuhad, A. Garg, and K. Chopra. 2009. Probiotics: Delineation of prophylactic and therapeutic benefits. J. Med. Food 12:219-235. http://dx.doi.org/10.1089/jmf.2007.0544.

Kawase, M., H. Hashimoto, M. Hosoda, H. Morita, and A. Hosono. 2000. Effect of administration of fermented milk containing whey protein concentrate to rats and healthy men on serum lipids and blood pressure. J. Dairy Sci. 83:255-263.

Kiessling, G., J. Schneider, and G. Jahreis. 2002. Long-term consumption of fermented dairy products over 6 months increases HDL cholesterol. Eur. J. Clin. Nutr. 56:843-849.

Kneeman, J. M., J. Misdraji, and K. E. Corey. 2012. Secondary causes of nonalcoholic fatty liver disease. Therap. Adv. Gastroenterol. 5:199-207. http://dx.doi.org/10.1177/1756283X11430859.

Kumar, M., R. Nagpal, R. Kumar, R. Hemalatha, V. Verma, A. Kumar, C. Chakraborty, B. Singh, F. Marotta, Sh. Jain, and H. Yadav. 2012. Cholesterol-lowering probiotics as potential biotherapeutics for metabolic diseases. Exp. Diabetes Res. 2012:902917: http://dx.doi.org/10.1155/2012/902917.

Lata, J., J. Jurankova, M. Kopacova, and P. Vitek. 2011. Probiotics in hepatology. World J. Gastroenterol. 17:2890-2896. http://dx.doi. org/10.3748/wjg.v17.i24.2890.

Lewis, S. J., and S. Burmeister. 2005. A double-blind placebo-controlled study of the effects of Lactobacillus acidophilus on plasma lipids. Eur. J. Clin. Nutr. 59:776-780.

Li, Z., S. Yang, H. Lin, J. Huang, P. A. Watkins, A. B. Moser, C. Desimone, X. Y. Song, and A. M. Diehl. 2003. Probiotics and antibodies to TNF inhibit inflammatory activity and improve nonalcoholic fatty liver disease. Hepatology 37:343-350.

Loguercio, C., T. De Simone, A. Federico, F. Terracciano, C. Tuccillo, M. Di Chicco, and M. Cartenì. 2002. Gut-liver axis: A new point of attack to treat chronic liver damage? Am. J. Gastroenterol. 97:2144-2146.

Lomonaco, R., N. E. Sunny, F. Brill, and K. Cusi. 2013. Nonalcoholic fatty liver disease: Current issues and novel treatment approaches. Drugs 73:1-14. http://dx.doi.org/10.1007/s40265-012-0004-0.

Lye, H. S., C. Y. Kuan, J. A. Ewe, W. Y. Fung, and M. T. Liong. 2009. The improvement of hypertension by probiotics: Effects on cholesterol, diabetes, renin, and phytoestrogens. Int. J. Mol. Sci. 10:3755-3775. http://dx.doi.org/10.3390/ijms10093755.

Malaguarnera, M., F. Greco, G. Barone, M. P. Gargante, M. Malaguarnera, and M. A. Toscano. 2007. Bifidobacterium longum with fructo-oligosaccharide (FOS) treatment in minimal hepatic encephalopathy: A randomized, double-blind, placebo controlled study. Dig. Dis. Sci. 52:3259-3265.

Malaguarnera, M., M. Vacante, T. Antic, M. Giordano, G. Chisari, R. Acquaviva, S. Mastrojeni, G. Malaguarnera, A. Mistretta, G. Li Volti, and F. Galvano. 2012. Bifidobacterium longum with fructooligosaccharides in patients with non alcoholic steatohepatitis. Dig. Dis. Sci. 57:545-553. http://dx.doi.org/10.1007/s10620-011$1887-4$.

Metchnikoff, I. I. 1907. The Prolongation of Life: Optimistic Studies. Translated and edited by P. Chalmers Mitchell. Heinemann, London, UK. Reprinted (2004) by Springer Publishing Co., New York, NY.

Nanda, K. 2004. Non-alcoholic steatohepatitis in children. Pediatr. Transplant. 8:613-618.

Naruszewicz, M., M. L. Johansson, D. Zapolska-Downar, and H. Bukowska. 2002. Effect of Lactobacillus plantarum 299v on cardiovascular disease risk factors in smokers. Am. J. Clin. Nutr. $76: 1249-1255$.

Nguyen, T. D., J. H. Kang, and M. S. Lee. 2007. Characterization of Lactobacillus plantarumph04, a potential probiotic bacterium with cholesterol-lowering effects. Int. J. Food Microbiol. 113:358-361.

Niazmand, R., A. Niazmand, M. Sarabi, N. Arabporiani, and A. Doaei. 2010. Effect of bioyogurt consumption on fatty metabolites of serum and colonic microflora in healthy subjects. J. Agric. Sci. Technol. 12:597-603.

Ooi, L.-G., and M.-T. Liong. 2010. Cholesterol-lowering effects of probiotics and prebiotics: A review of in vivo and in vitro findings. Int. J. Mol. Sci. 11:2499-2522. http://dx.doi.org/10.3390/ ijms11062499.

Paik, H. D., J.-S. Park, and E. Park. 2005. Effects of Bacillus polyfermenticus SCD on lipid and antioxidant metabolisms in rats fed a high-fat and high-cholesterol diet. Biol. Pharm. Bull. 28:12701274 .

Pocock, S. J. 1983. Clinical Trials: A Practical Approach. John Wiley \& Sons, New York, NY.

Rafiq, N., and Z. Younossi. 2009. Nonalcoholic fatty liver disease: A practical approach to evaluation and management. Clin. Liver Dis. 13:249-266. http://dx.doi.org/10.1016/j.cld.2009.02.009.

Roberts, E. A. 2005. Non-alcoholic fatty liver disease (NAFLD) in children. Front. Biosci. 10:2306-2318.

Sadrzadeh-Yeganeh, H., I. Elmadfa, A. Djazayery, M. Jalali, R. Heshmat, and M. Chamary. 2010. The effects of probiotic and conventional yoghurt on lipid profile in women. Br. J. Nutr. 103:17781783. http://dx.doi.org/10.1017/S0007114509993801.

Viana, J. V., A. G. Da Cruz, S. S. Zoellner, R. Silva, and A. L. D. Batista. 2007. Probiotic foods: Consumer perception and attitudes. Int. J. Food Sci. Technol. 43:1577-1580. http://dx.doi. org/10.1111/j.1365-2621.2007.01596-x.

Wang, Y., N. Xu, A. Xi, Z. Ahmed, B. Zhang, and X. Bai. 2009. Effects of Lactobacillus plantarum MA2 isolated from Tibet kefir on lipid metabolism and intestinal microflora of rats fed on highcholesterol diet. Appl. Microbiol. Biotechnol. 84:341-347. http:// dx.doi.org/10.1007/s00253-009-2012-x.

Wong, V. 2008. Recent advances in the management of nonalcoholic fatty liver disease. Med. Bull. 13:19-22.

Xiao, J. Z., S. Kondo, N. Takahashi, K. Miyaji, K. Oshida, A. Hiramatsu, K. Iwatsuki, S. Kokubo, and A. Hosono. 2003. Effects of milk products fermented by Bifidobacterium longum on blood lipids in rats and healthy adult male volunteers. J. Dairy Sci. 86:2452-2461.

Yadav, H., S. Jain, and P. R. Sinha. 2008. Oral administration of dahi containing probiotic Lactobacillus acidophilus and Lactobacillus casei delayed the progression of streptozotocin-induced diabetes in rats. J. Dairy Res. 75:189-195. http://dx.doi.org/10.1017/ S0022029908003129.

Zar, J. H. 1998. Biostatistical Analysis. Pearson Press, New York, NY. Zhuang, G., X.-M. Liu, Q.-X. Zhang, F.-W. Tian, H. Zhang, H.-P. Zhang, and W. Chen. 2012. Research advances with regards to clinical outcome and potential mechanisms of the cholesterol-lowering effects of probiotics. Clin. Lipidol. 7:501-507. http://dx.doi. org/10.2217/clp.12.40. 